\title{
Universal correspondence between edge spin accumulation and equilibrium spin currents in nanowires with spin-orbit coupling
}

\author{
I. V. Tokatly, ${ }^{1,2,3}$ B. Bujnowski, ${ }^{3, *}$ and F. S. Bergeret ${ }^{4,3, \dagger}$ \\ ${ }^{1}$ Nano-Bio Spectroscopy Group, Departamento Física de Materiales, Universidad del País Vasco, \\ Avenida Tolosa 72, E-20018 San Sebastián, Spain \\ ${ }^{2}$ IKERBASQUE, Basque Foundation for Science, E-48011 Bilbao, Spain \\ ${ }^{3}$ Donostia International Physics Center, Manuel de Lardizabal 4, E-20018 San Sebastián, Spain \\ ${ }^{4}$ Centro de Física de Materiales (CFM-MPC), Centro Mixto CSIC-UPV/EHU, Manuel de Lardizabal 5, \\ E-20018 Donostia-San Sebastian, Basque Country, Spain
}

(Received 23 January 2019; revised manuscript received 23 September 2019; published 19 December 2019)

\begin{abstract}
Although equilibrium spin currents (ESCs) may exist in materials with spin-orbit coupling, they are not transport currents and cannot lead to spin accumulation in the presence of time-reversal symmetry. It is for this reason that the detection of ESCs has remained elusive. Here we show that in a nanowire with spin-orbit coupling, breaking the time-reversal symmetry by a Zeeman field leads to a bulk equilibrium spin current which manifests itself in a sizable edge spin polarization, transverse to the Zeeman field. The net accumulated spin does not depend on specific properties of the wire ends, being fully determined by the bulk spin current. This bulk-boundary correspondence is a universal property that occurs in both the normal and superconducting states independently of the degree of disorder. The transverse edge spin polarization is strongly enhanced in the superconducting state when the Zeeman energy is of the order of the induced superconducting gap. This leads to a hitherto unknown transverse magnetic susceptibility that can be much larger than the longitudinal, and it drastically changes the paramagnetic response of the nanowire.
\end{abstract}

DOI: $10.1103 /$ PhysRevB.100.214422

\section{INTRODUCTION}

Spin currents have been the subject of intensive investigations in several branches of condensed-matter physics [1-4]. In 2003 Rashba himself demonstrated that any system described by the standard two-dimensional Rashba Hamiltonian may support spin currents even in thermodynamical equilibrium [5]. Such equilibrium spin currents (ESCs) in materials with spin-orbit coupling (SOC) have attracted a great deal of attention [2,6-10]. Spin currents are usually detected indirectly by, for example, measuring the spin voltage with a ferromagnetic probe [11] or spin-Hall magnetoresistance [12], induced by nonequilibrium spin accumulation. However, ESCs do not lead to such spin accumulation and therefore cannot be detected by these methods. Indeed, ESCs, being background nontransport currents, appear to be conceptually unobservable, although some proposals relating them to a detectable mechanical torque have been put forward $[8,9]$. For these reasons, the interpretation of the ESC remained under debate. In addition, in the presence of SOC the spin is not conserved in a customary sense. Theoretically, the latter controversy can be removed by treating the SOC as an external SU(2) gauge field [13,14], but from the experimental point of view ESCs still remain elusive.

\footnotetext{
*bogusz.bujnowski@gmail.com

${ }^{\dagger}$ fs.bergeret@csic.es
}

Here we demonstrate a correspondence between ESCs in nanowires with SOC and a transverse spin polarization induced at the edges of the wires, as schematically shown in Fig. 1. Contrary to ESCs, spin polarization can be experimentally detected. The correspondence we found is universal in the sense that it holds for any (quasi)one-dimensional manybody system, provided the particle-particle interaction is spin independent. Specifically, ESCs appear when a magnetic field $B$ with a component perpendicular to the SOC is applied, inducing a Zeeman splitting field, $h=g \mu_{B} B$. We show that this bulk ESC is always accompanied by an edge spin accumulation that is transverse to both the Zeeman field and SOC. This equilibrium transverse spin accumulation can also be understood as a redistribution of the spin density in the system in response to a dc field. Hence, a measurement of the transverse spin density would be unequivocal evidence of ESCs in nanowires. Interestingly, this transverse edge spin accumulation shows up not only in the normal state [15] but also when the nanowire has a superconducting gap $\Delta$ in its density of states induced, for example, by the proximity to a superconductor. In other words, we find a connection between the existence of ESCs and an anomalous paramagnetic response of a superconductor that generalizes the well-established theory of the Knight shift in superconductors [16-18].

Semiconducting nanowires with spin-orbit coupling, as InAs and InSb, contacted to conventional superconductors have been intensively explored in recent years, mainly due to the possibility of creating Majorana zero modes and 


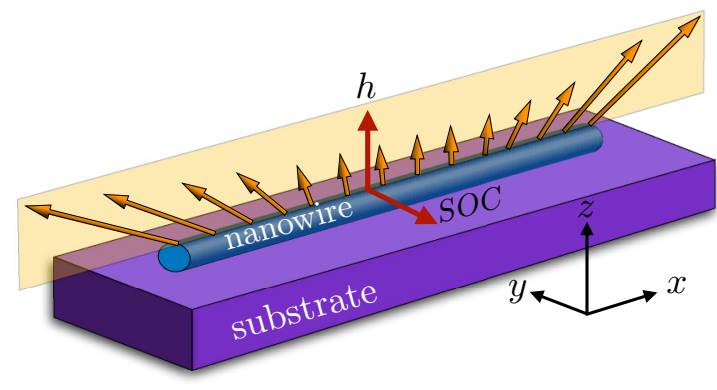

FIG. 1. Schematic view of the system under consideration: a nanowire with spin-orbit coupling in a Zeeman field perpendicular to the substrate. Due to the existence of an equilibrium spin current a transverse-to-the-field component of the magnetization, localized at the edges, is induced. The orange arrows represent the total induced spin. Its transverse component has the opposite sign at both edges of the wire

topological superconductivity in these systems [19-31]. Interestingly, both the ESCs and Majorana zero modes require a component of the Zeeman field perpendicular to the SOC. In the context of Majorana fermions, several theoretical works have made a connection between the spin polarization induced at the edges of the wire and the topological transition when $h=\sqrt{\mu^{2}+\Delta^{2}}$, where $\mu$ is the chemical potential of the wire [25,32-34]. Our work shows that the transverse edge spin polarization is a universal property of nanowires supporting ESCs and it exists at all values of $h$, including those far below the topological transition, and in both normal and superconducting states. Therefore, its detection cannot be associated with Majorana zero modes [15,25] but can be associated, as we will see, with the existence of ESCs.

Specifically, we show that as a function of the Zeeman field $h$, the total spin accumulation shows, in general, a cusp at $h=\sqrt{\mu^{2}+\Delta^{2}}$. Interestingly, when $\Delta \ll \mu$, the transverse spin accumulation shows, in addition, a sharp maximum at $h \approx \Delta$ and can be much larger than the magnitude at the cusp when $h=\mu$. We analyze in detail this maximum of the spin accumulation and show its robustness against disorder. Finally, we present analytical results for the spatial distribution of the magnetic moment induced as a response to the Zeeman field. We find that the transverse susceptibility close to the edge of the wire can be much larger than the longitudinal one for small values of the SOC. It is worth noticing that all our results have been obtained analytically by using different methods in the different analyzed situations. Our different ways of solving the problem, in principle, can be used and generalized for more complex geometries and materials.

\section{BULK BOUNDARY CORRESPONDENCE}

First, we demonstrate the universality of the correspondence between ESCs and the transverse spin accumulation in one-dimensional (1D) systems using general principles. We consider the setup shown in Fig. 1, where a nanowire is deposited on a substrate. The wire has an intrinsic SOC which is assumed to be linear in momentum and determined by the symmetry of the structure. In addition, a Zeeman/exchange field $h$ is applied perpendicular to the substrate and to the
SOC. Without loss of generality let the wire be oriented along the $x$ axis, Rashba SOC be oriented along the $y$ axis, and a Zeeman field be oriented along the $z$ axis. The many-body Hamiltonian modeling the system reads

$$
H=\sum_{n=1}^{N}\left[\frac{\hat{\mathbf{p}}_{n}^{2}}{2 m}+\alpha \hat{p}_{n}^{x} \sigma_{n}^{y}+h \sigma_{n}^{z}+V_{c}\left(\mathbf{r}_{n}^{\perp}, x_{n}\right)\right]+H_{\mathrm{int}},
$$

where the index $n$ labels the particles, $\hat{p}_{n}^{k}=-i \partial_{x_{n}^{k}}$, with $k=$ $x, y, z$, are components of the momentum operator of $n$th particle, $\alpha$ is the strength of the SOC, $V_{c}\left(\mathbf{r}^{\perp}, x\right)$ is a confinement potential, $\mathbf{r}^{\perp}=(y, z)$, and $H_{\text {int }}$ describes a spin-independent interaction.

The main observables we are interested here are the spin density $\mathbf{s}=\left(s^{x}, s^{y}, s^{z}\right)$ and components of the spin current density $\mathbf{j}_{k}=\left(j_{k}^{x}, j_{k}^{y}, j_{k}^{z}\right)$. By defining the spin current operator in the standard way $[2,8,10,13]$,

$$
\hat{j}_{k}^{a}(\mathbf{r})=\frac{1}{4} \sum_{n=1}^{N}\left\{\left\{\frac{\partial H}{\partial \hat{p}_{n}^{k}}, \sigma_{n}^{a}\right\}, \delta\left(\mathbf{r}-\mathbf{r}_{n}\right)\right\},
$$

and using the Hamiltonian (1) we obtain the following equation of motion for the spin density [13]:

$$
\partial_{t} \mathbf{s}+\partial_{r^{k}} \mathbf{j}_{k}+\alpha \hat{\mathbf{y}} \times \mathbf{j}_{x}+h \hat{\mathbf{z}} \times \mathbf{s}=0 .
$$

Here the third term is the spin-orbit torque, while the last term is the usual spin torque due to the Zeeman field, which leads to the spin Hanle effect [11].

Let us now concentrate on the equilibrium state. The Hamiltonian in (1) is real, and therefore, its eigenfunctions can be chosen to be real. This implies that in equilibrium there is no component of the spin density parallel to the SOC, $s^{y}(\mathbf{r})=0$. Similarly, we find that the system can support only an equilibrium spin current $j_{k}^{y}(\mathbf{r})$ polarized along the $y$ axis. Therefore, in equilibrium the spin-orbit torque vanishes, and the $y$ component of the spin continuity equation (3) takes the form

$$
\partial_{r^{k}} j_{k}^{y}(\mathbf{r})+h s^{x}(\mathbf{r})=0 .
$$

Finally, by integrating this equation over the wire cross section we simplify it as follows:

$$
\partial_{x} J_{x}^{y}(x)+h S^{x}(x)=0,
$$

where $\mathbf{S}(x)$ is the line spin density along the wire and $J_{x}^{y}(x)$ is the ESC flowing through the wire cross section. Equation (5) shows that ESC in the bulk of the system may exist only if the $x$ component of the spin is accumulated at the edges. In other words, Eq. (5) establishes a correspondence between the bulk property of the system, the ESC, and the net spin accumulation transverse to the Zeeman field at the edge of a finite system. An example of this correspondence was obtained in Ref. [15] for a noninteracting finite 1D wire contacted to leads.

We consider a semi-infinite wire with the external potential $V_{c}$ in Eq. (1) confining the electrons in the right half-space at $x>0$ in such a way that all occupied states decay exponentially at $x \rightarrow-\infty$. In this case the spin accumulation at the edge around $x=0$ is obtained by integrating Eq. (5),

$$
\bar{S}^{x} \equiv \int_{-\infty}^{+\infty} d x S^{x}(x)=-\frac{1}{h} J_{x}^{y}(\infty)
$$



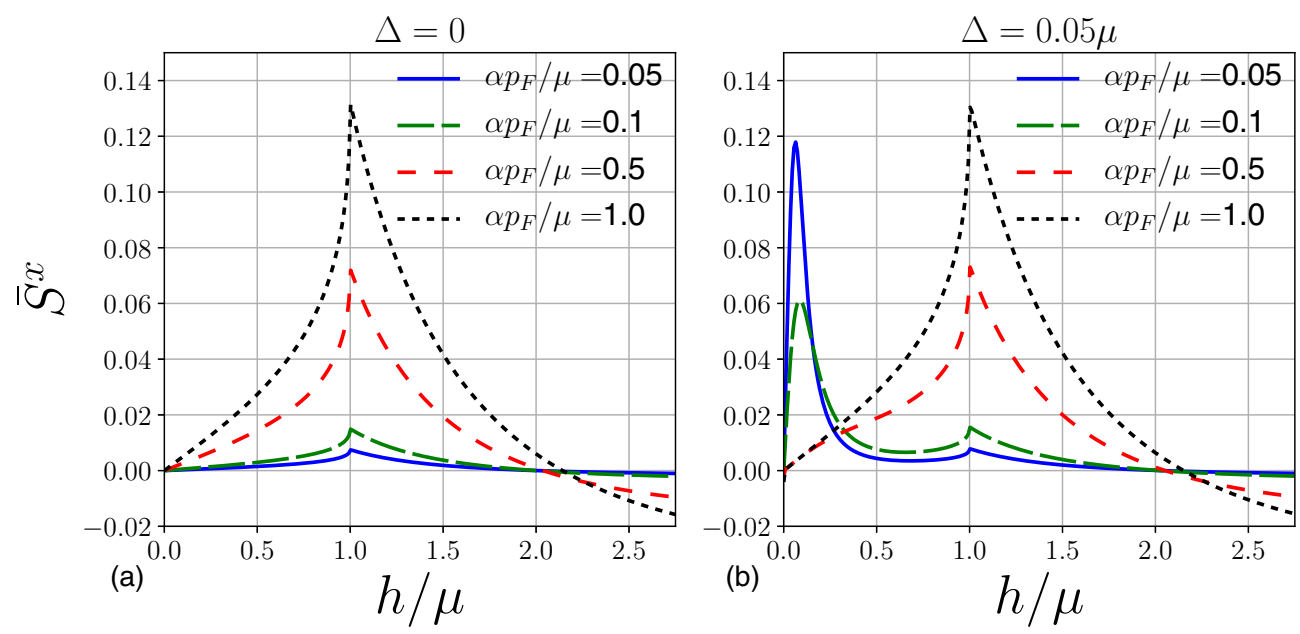

FIG. 2. Transverse spin accumulation as a function of the Zeeman field $h=g \mu_{B} B$ for various values of the SOC strength $\alpha p_{F}$ in the (a) normal and (b) superconducting cases at $T=0$.

This equation is of particular interest: On the one hand, it connects the ESC on the right-hand side, which is a rather theoretical object [5], with a measurable quantity, the edge spin polarization (left-hand side). On the other hand, it provides a direct way to determine the edge spin accumulation by calculating a bulk property, the ESC, independently of boundary conditions. In terms of physics, Eq. (5) describes a noncollinear redistribution of the spin density along the wire as a response to a static and homogeneous Zeeman field. Clearly, for a finite wire, the transverse component of the spin at opposite edges is equal in magnitude but oriented in the opposite direction, as shown schematically in Fig. 1. In the following we use Eq. (6) to compute the transversal spin accumulation in normal and superconducting wires.

\section{BALLISTIC NORMAL AND SUPERCONDUCTING WIRES}

As a first example we consider a one-channel normal ballistic wire described by the 1D version of the Hamiltonian in Eq. (1) with $H_{\text {int }}=V_{c}=0$ [15]. The spin current can be written as

$$
J^{y} \equiv J_{x}^{y}=\int \frac{d p}{2 \pi} \operatorname{Tr}\left[\sigma^{y} \hat{v} \hat{\rho}_{p}\right],
$$

with the velocity operator $\hat{v}=\frac{\partial H}{\partial \hat{p}}=\frac{p}{m}+\alpha \hat{\sigma}^{y}, p=p_{x}$, and the equilibrium density matrix written in terms of the projectors on the two eigenstates $E_{p}^{ \pm}=\xi_{p} \pm E_{p}$,

$$
\hat{\rho}_{p}=\sum_{i= \pm} \rho_{p}^{i} f\left(E_{p}^{i}\right), \rho_{p}^{ \pm}=\frac{1}{2}\left(\mathbb{1} \pm \frac{\alpha p \hat{\sigma}^{y}+h \hat{\sigma}^{z}}{E_{p}}\right),
$$

where $\xi_{p}=p^{2} / 2 m-\mu, E_{p}=\sqrt{\alpha^{2} p^{2}+h^{2}}$, and $f(E)$ is the Fermi distribution function. We focus on the case $T=0$ and $\mu, \alpha, h>0$. Introducing the Fermi momenta for the two spin split bands

$$
p^{ \pm}=\sqrt{2 m\left[m \alpha^{2}+\mu \mp \sqrt{\left(m \alpha^{2}+\mu\right)^{2}+h^{2}-\mu^{2}}\right]},
$$

the current can be expressed as the sum of the contributions from the two bands:

$$
J^{y}=J_{-}^{y}+\Theta(\mu-h) J_{+}^{y},
$$

where + and - label the upper and lower bands, respectively. The two contributions are

$$
\begin{aligned}
J_{ \pm}^{y}= & \frac{\alpha p^{ \pm}}{\pi} \pm \frac{1}{2 m \pi}\left[\frac{p^{ \pm}}{\alpha} \sqrt{\left(\alpha p^{ \pm}\right)^{2}+h^{2}}\right. \\
& \left.-\frac{h^{2}}{\alpha^{2}} \ln \left(\sqrt{\frac{\left(\alpha p^{ \pm}\right)^{2}}{h^{2}}+1}+\frac{\alpha}{h} p^{ \pm}\right)\right] .
\end{aligned}
$$

Clearly, the ESC is finite only if both the SOC and Zeeman field are finite. The transverse spin accumulation can be computed by substituting Eq. (11) into Eq. (6). The result is shown in Fig. 2(a). By increasing $h$ from zero towards $\mu$, the spin current increases monotonically. At $h=\mu, p^{+}=0$, and for larger values of $h$ the upper band does not contribute to the current. This results in a cusplike maximum in the spin current, also obtained in Ref. [15] for a normal wire [35].

Now we assume that the wire is placed on top of a superconductor so that it is fully proximitized. As is customary, the system is described by the Bogoliubov-de Gennes (BdG) Hamiltonian, which can be obtained from Eq. (1) after extension to the Nambu-spin space:

$$
\mathcal{H}=\left(\frac{\hat{p}^{2}}{2 m}-\mu+\alpha \hat{p} \hat{\sigma}^{y}\right) \tau_{z}+h \hat{\sigma}^{z}+\Delta \tau_{x},
$$

where $\Delta$ is the SC order parameter and $\tau_{j}$ are the Pauli matrices in the Nambu space. This Hamiltonian leads to a four-band spectrum,

$$
E_{ \pm i}= \pm \sqrt{\xi_{p}^{2}+(\alpha p)^{2}+h^{2}+\Delta^{2}+(-1)^{i} 2 Q},
$$

with $i=1,2$ and $Q=\sqrt{\left(\xi_{p}^{2}+\Delta^{2}\right) h^{2}+\xi_{p}^{2} \alpha^{2} p^{2}}$. In this case the spin current can be written as

$$
J^{y}=\int \frac{d p}{2 \pi} \sum_{i= \pm 1, \pm 2} \operatorname{Tr}\left[P_{i} \hat{J}_{x}^{y}\right] f\left(E_{i}\right),
$$



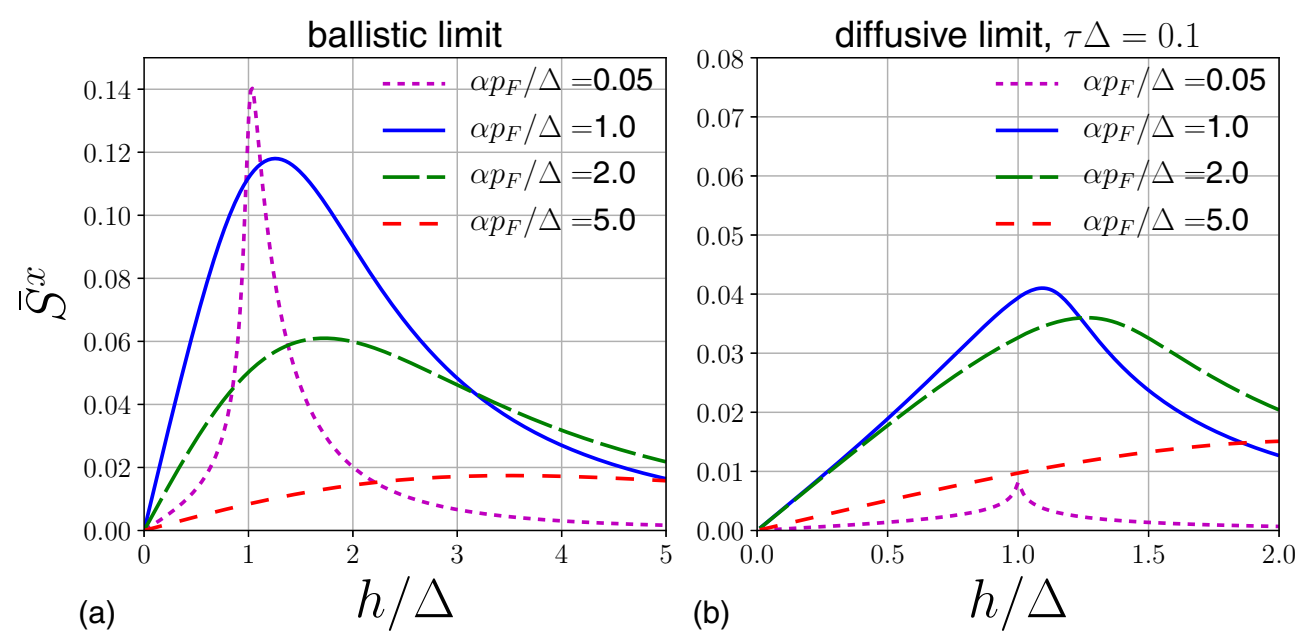

FIG. 3. Spin accumulation calculated in the quasiclassical limit as a function of the Zeeman field for various values of $\alpha p_{F}$ in the (a) ballistic and (b) diffusive limits at $T=0$. In the diffusive limit we have chosen $\Delta \tau=0.1$.

where $P_{i}=\prod_{j \neq i} \frac{\mathcal{H}-E_{j}}{E_{i}-E_{j}}$ is the projection operator to the eigenstate $\left|n_{i}\right\rangle$ of the BdG Hamiltonian and $\hat{J}_{x}^{y}=\left(\frac{p}{m} \hat{\sigma}^{y}+\alpha\right) \tau_{z}$ is the spin current operator written in Nambu-spin space. Explicit evaluation of this expression at $T=0$ gives

$$
\begin{aligned}
J^{y}= & \frac{\alpha}{2 \pi} \int d p\left\{1-\frac{\xi_{p}}{2} \sum_{i=1,2}\left[\frac{1}{E_{i}}\left(1+(-1)^{i} \frac{E_{p}^{2}}{Q}\right)\right]\right\} \\
& -\frac{\alpha}{2 \pi} \int d p\left\{\frac{p^{2}}{2 m} \sum_{i=1,2}\left[\frac{1}{E_{i}}\left(1+(-1)^{i} \frac{\xi_{p}^{2}}{Q}\right)\right]\right\} .
\end{aligned}
$$

It is important to note that this expression is rather general and valid for arbitrary values of the parameters. The numerical evaluation of the integral can be performed straightforwardly and shows two prominent features, as seen in Fig. 2(b). The first one is the same cusplike local maximum, as in the normal case, which now appears at $h=\sqrt{\mu^{2}+\Delta^{2}}$. This value corresponds to the critical Zeeman field, above which the wire is in the topological phase. It is worth mentioning that this cusplike feature is obtained, practically, over the whole range of parameters. Only when $\mu \rightarrow 0$ and $m \alpha^{2} \gg \Delta$ does the transverse spin not show a maximum at $h=\Delta$ and the discontinuity in the slope of $S^{x}$ at $h=\Delta$ is smoothed out [34]. In this work we are not interested in the zero-chemicalpotential limit and focus on cases when $\mu \gg \Delta, m \alpha^{2}$.

The second prominent feature, as seen from Fig. 2(b), is the maximum that appears at values of $h \ll \mu$ and that for small enough values of $\alpha$ is located at $h \approx \Delta$. Interestingly, by lowering $\alpha$ the peak first becomes narrow and simultaneously increases in magnitude, exceeding by far the local maximum at the topological transition. A further decrease of $\alpha$ reduces the height of the peak until the spin current and thus the spin accumulation vanish when $\alpha \rightarrow 0$. Since this maximum in the spin accumulation occurs at values of the fields much smaller than $\mu$, we can investigate the behavior of the transverse spin accumulation at small $\alpha$ within the quasiclassical approach
[36]. The spin current within this approach is given by [37]

$$
J_{x}^{a}=\frac{i \pi N_{0}}{2} T \sum_{\omega_{n}} \operatorname{Tr}\left\langle v_{F} \hat{\sigma}^{a} \breve{g}\right\rangle .
$$

Here $\check{g}$ is the quasiclassical Green's function that is obtained from the Gor'kov Green's function by the $\xi$ integration [36] $\check{g}=i / \pi \int d \xi \breve{G}\left(p, \omega_{n}\right),\langle\cdots\rangle$ is the average over the Fermi surface, $T$ is the temperature, $N_{0}=1 /\left(2 \pi v_{F}\right)$ is the $1 \mathrm{D}$ density of states at the Fermi level, $\omega_{n}=(2 n+1) \pi T$ are the Matsubara frequencies, and $v_{F}$ and $p_{F}$ denote the Fermi velocity and Fermi momentum, respectively. At $T \rightarrow 0$ we obtain, for the spin current,

$$
J^{y}=\int \frac{d \omega}{\pi} \operatorname{Im}\left[\frac{\alpha p_{F}\left(\omega^{2}+\Delta^{2}+i R\right)}{R \sqrt{\omega^{2}+\Delta^{2}-h^{2}-\alpha^{2} p_{F}^{2}+2 i R}}\right],
$$

with $R=\sqrt{h^{2} \omega^{2}+\left(\alpha p_{F}\right)^{2}\left(\omega^{2}+\Delta^{2}\right)}$. From this expression and Eq. (6) we compute the dependence $S^{x}(h)$ around $h=\Delta$, which is shown in Fig. 3(a). As expected, in this range of parameters, the quasiclassical result agrees with the exact calculation [see Fig. 2(b)]. In particular, the peak is more pronounced for small values of SOC and washes out with increasing SOC strength. At $h=\Delta$ and for $\alpha p_{F} \ll \Delta$ the integral can be approximated by keeping only leading-order terms in $\alpha / \Delta$ (see Appendix A for details),

$$
J^{y} \approx-\frac{\alpha p_{F}}{\pi} \int_{0}^{\infty} d \omega \frac{\Delta^{2}}{R^{3 / 2}} \approx-2 \sqrt{\frac{\alpha p_{F} \Delta}{\pi}} \frac{\Gamma(5 / 4)}{\Gamma(3 / 4)},
$$

where we approximate $R \approx \sqrt{(\omega \Delta)^{2}+\left(\alpha p_{F}\right)^{2}}$. In a similar way, we can also determine the spin current at $h=\Delta$ for finite temperature and $\alpha p_{F} \ll T \ll \Delta$ (see Appendix A for details),

$$
J^{y} \approx-\frac{\alpha p_{F}}{\pi^{\frac{3}{2}}} \sqrt{\frac{\Delta}{T}} \sum_{n=0}^{\infty} \frac{1}{(2 n+1)^{\frac{3}{2}}} .
$$




\section{DIFFUSIVE SUPERCONDUCTING WIRE}

So far we have considered a pure ballistic situation. It is, however, known that $s$-wave superconductivity is insensitive to elastic disorder, and therefore, one would expect to obtain qualitatively similar results for a disorder superconducting nanowire supporting ESCs. Notice that in this case the wire is a multichannel wire described by Hamiltonian (1). For the sake of comparison with the ballistic single-channel results we calculate the observables normalized to a single conduction channel. This essentially means that in the expressions for the observables below we use the 1D density of states $N_{0}$ as in the previous sections. To estimate the measurable spin one should multiply the obtained result by the number of conducting channels, which is approximately given by $p_{F}^{2} \mathcal{S} / 4 \pi$, where $\mathcal{S}$ is the cross section of the wire. In the diffusive limit the lateral dimensions of the wire are much shorter than the superconducting coherence length but larger than the elastic mean free path. In this case the spin current can be written in terms of the isotropic in momentum quasiclassical Green's function $\breve{\mathfrak{g}}$ [38],

$$
J^{y}=-i \frac{\pi D}{2} N_{0} T \sum_{\omega_{n}} \operatorname{Tr}\left\{\hat{\sigma}^{y} \check{\mathfrak{g}}_{x} \tilde{\nabla}_{x} \mathfrak{\mathfrak { g }}\right\}
$$

where $D=v_{F}^{2} \tau$ is the diffusion constant, $\tau$ is the momentum relaxation time, $\tilde{\nabla}_{x} \cdot=\partial_{x} \cdot-i\left(\kappa_{\alpha} / 2\right)\left[\hat{\sigma}^{y}, \cdot\right]$, and $\kappa_{\alpha}=2 m \alpha$ is the inverse of the spin precession length. To find $\mathfrak{g}$ we solve the Usadel equation for a bulk system [38],

$$
\frac{1}{2}[\hat{\Gamma} \check{\mathfrak{g}}, \check{\mathfrak{g}}]-\left[\left(\hat{\omega}_{n}-i h \hat{\sigma}_{z}\right) \tau_{3}+\Delta \tau_{1}, \check{\mathfrak{g}}\right]=0,
$$

where $\hat{\Gamma} \check{\mathfrak{g}}=\left[\hat{\sigma}^{y},\left[\hat{\sigma}^{y}, \check{\mathfrak{g}}\right]\right] / 4 \tau_{\alpha}$ and $1 / \tau_{\alpha}=D \kappa_{\alpha}^{2}$ is the inverse Dyakonov-Perel spin relaxation time. The Green's function has to fulfill the normalization condition $\check{\mathfrak{g}}^{2}=\mathbb{1}$.

The matrix structure of the quasiclassical Green's functions entering the Usadel equation is $\check{\mathfrak{g}}=\hat{\mathfrak{g}} \tau_{3}+\hat{\mathfrak{f}} \tau_{1}$, and for a Zeeman field in the $z$ direction the matrices $\hat{\mathfrak{g}}$ and $\hat{\mathfrak{f}}$ are diagonal in the spin space with components $\mathfrak{g}^{ \pm}$and $\mathfrak{f}^{ \pm}$, respectively. At $T=0$ the expression for the equilibrium spin current, Eq. (20), reduces to

$$
J^{y}=-\frac{\alpha p_{F}}{\Delta} \Delta \tau \int \frac{d \omega}{\pi}\left(1-\mathfrak{g}^{+} \mathfrak{g}^{-}-\mathfrak{f}^{+} \mathfrak{f}^{-}\right) .
$$

Substituting in this equation the solution of Eq. (21) and after using our correspondence, Eq. (6), one obtains the transverse spin accumulation as shown in Fig. 3(b). Thus, also in the diffusive limit we find a maximum $S^{x}$ at $h \approx \Delta$ for small values of SOC.

In the limit $T \ll 1 / \tau_{\alpha} \ll h \sim \Delta$ we find that, within logarithmic accuracy, the spin current of Eq. (22) is given by

$$
J^{y} \approx \frac{2}{3} \frac{\alpha p_{F}}{\pi} \Delta \tau \ln \left(\Delta \tau_{\alpha}\right) .
$$

Thus, in the diffusive case the peak in the spin current at $h=$ $\Delta$ for small values of the SOC is reduced by a factor $\sim \delta \ln \delta$, with $\delta=\Delta \tau \sqrt{\frac{\alpha p_{F}}{\Delta}} \ll 1$, with respect to the pure ballistic case [see Eq. (18)]. This explains the difference between the peak heights in Figs. 3(a) and 3(b) for $p_{F} \alpha=0.05 \Delta$. Notice, however, that this difference is not that pronounced for larger values of the SOC.

\section{SPATIAL DEPENDENCE OF THE SPIN SUSCEPTIBILITY IN A FINITE WIRE}

Previously, we used the general expression (6) to relate the bulk ESC to the transverse spin polarization $\bar{S}^{x}$ in a finite wire (see Fig. 1). We understand that $\bar{S}^{x}$ accumulates at the edge and decays from the edge towards the bulk, but the exact spatial distribution has to be determined in each particular case. In the normal state the dependence $S^{x}(x)$ was computed numerically in Ref. [15] for a wire connected to two leads and for values of $h$ of the order of $|\mu|$. Here we focus on the superconducting state in the relevant quasiclassical regime, $h \ll \mu$, for which the Eilenberger equation holds,

$$
\pm v_{F} \partial_{x} \check{g}_{ \pm}= \pm i \alpha p_{F} \hat{\sigma}^{y}+i \tau_{3}\left(i \omega_{n}+h \hat{\sigma}^{z}\right)+i \tau_{2} \Delta, \check{g}_{ \pm} .
$$

Here $\breve{g}_{ \pm}$denotes the Green's functions for both propagation directions $\pm v_{F}$. We assume that the wire extends over the region $x>0$. At the edge, $x=0$, no current flows, and the boundary condition imposes that $\breve{g}_{+}(0)=\breve{g}_{-}(0)$. We focus here on the spin susceptibility, i.e., the magnetic linear response of the system in the presence of a small Zeeman field. In other words we compute the spatial dependence of the spin in linear order in $h$. To this end we perform perturbation theory and write the solution of Eq. (24) as $\breve{g}_{ \pm}(x)=\breve{g}_{B C S}+\delta \check{g}_{ \pm}(x)$, where $\check{g}_{B C S}=\left(\omega_{n} \tau_{3}+\Delta \tau_{2}\right) / E$ and $E=\sqrt{\omega_{n}^{2}+\Delta^{2}}$. The spin density is then obtained from $\delta S^{j}=$ $\pi i T N_{0} \frac{1}{8} \sum_{\omega_{n}} \operatorname{Tr}\left[\tau_{3} \hat{\sigma}^{j} \delta \breve{g}^{s}\right]$, where $\delta \check{g}^{s}=\delta \check{g}_{+}+\delta \check{g}_{-}$. Specifically, we obtain

$$
\delta \breve{g}^{S}=\frac{2 h \Delta \check{g}_{B C S} \tau_{1}}{E^{2}+\left(\alpha p_{F}\right)^{2}}\left[\hat{\sigma}^{z}+\frac{\alpha p_{F}}{E} e^{-\left(i \hat{\sigma}^{y} \kappa_{\alpha}+\frac{2 E}{v_{F}}\right) x}\right],
$$

and hence (see Appendix B for details),

$$
\begin{aligned}
& \frac{S^{x}}{S_{0}}=\Delta^{2} \pi T \sum_{\omega_{n}} \frac{\alpha p_{F} \cos \left(\kappa_{\alpha} x\right)}{E^{2}\left(E^{2}+\left(\alpha p_{F}\right)^{2}\right)} e^{-\frac{2 E x}{v_{F}}}, \\
& \frac{\delta S^{z}}{S_{0}}=\Delta^{2} \pi T \sum_{\omega_{n}} \frac{E+\alpha p_{F} \sin \left(\kappa_{\alpha} x\right) e^{-\frac{2 E x}{v_{F}}}}{E^{2}\left[E^{2}+\left(\alpha p_{F}\right)^{2}\right]},
\end{aligned}
$$

where $S_{0}=h N_{0}$ is the Pauli paramagnetic term. This is a remarkable result that shows that in a finite system, besides the longitudinal response $S^{z}=\delta S^{z}-S_{0}$, a finite transverse magnetization accumulated at the edge of the sample which decays toward the bulk over the superconducting coherence length. At low temperatures, the ratio $S^{x} / S^{z}$ at the edge of the sample, $x=0$, is proportional to $\Delta / \alpha p_{F}$, and therefore, the transverse magnetization can be much larger than $S^{z}$ provided $\alpha p_{F} \ll \Delta$. This result generalizes the well-established theory of paramagnetic response of superconducting systems with intrinsic SOC [18]. The full spatial dependence of $S^{x, z}(x)$ is shown in Fig. 4.

\section{SUMMARY}

We have demonstrated a universal correspondence between equilibrium spin currents and a transverse spin accumulation in wires with SOC and a perpendicular Zeeman field. Such spin accumulation appears in both normal and superconducting states. In the normal state the total edge spin is maximized for values of $h$ of the order of the chemical potential $\mu$. In the superconducting state the effect can be maximized at 


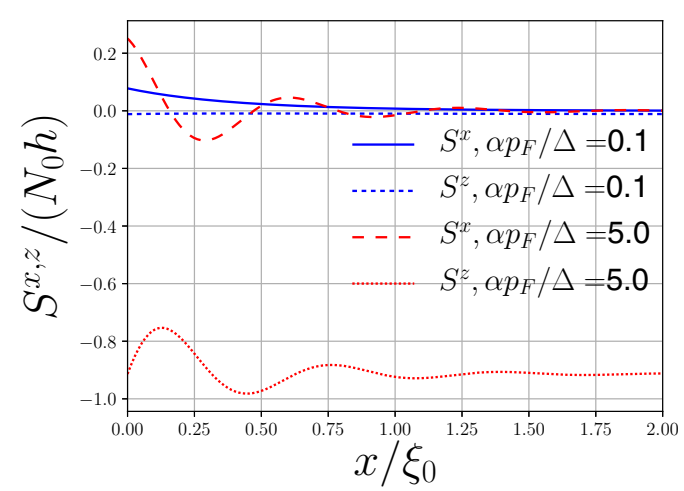

FIG. 4. Spatial dependence of the spin density parallel $\left(S^{z}\right)$ and perpendicular $\left(S^{x}\right)$ to the exchange field for a semi-infinite wire at $T=0$ and different values of SOC.

values of the SOC and Zeeman energy much smaller than $\mu$. We demonstrated that this effect is robust against disorder and manifest in a transverse magnetic susceptibility in finite systems.

A semiconductor nanowire with SOC in contact with a superconductor is a good candidate to observe the spin accumulation. If we use typical numbers for the wires used in Majorana experiments [21,27], $\alpha \sim 10^{-11} \mathrm{eV} \mathrm{m}$ and $p_{F} \sim$ $10^{8} \mathrm{~m}^{-1}$, we obtain $\alpha p_{F} \sim 10^{-3} \mathrm{eV}$. According to Fig. 3, the effect is maximized when $\alpha p_{F} / \Delta$ is of the order of unity, which is the case when a superconductor like $\mathrm{Nb}$, with $\Delta \sim$ $10^{-3} \mathrm{eV}$, is used. Notice that the results shown in all the figures refer to a single conduction channel, and hence, the edge magnetic moment is of the order of (or smaller than) a single electron spin, which in principle can be measured with stateof-the-art-techniques. However, a real nanowire has several conduction channels, and the measurable spin accumulation is estimated by multiplying the magnetic moment shown in all the figures by the number of channels. In particular, the diffusive limit [Fig. 3(b)] implies many channels, and hence, the size of the effect is comparable to the one in the pure ballistic case [Fig. 3(a)].

Measurements of the predicted transverse spin may require sensitive detection techniques. Since the transverse susceptibility predicted here is comparable in size to, or even larger than, the longitudinal one, customary measurements of the Knight shift using nuclear magnetic resonance may be suitable to reveal the existence of ESCs. Other recently developed techniques for local magnetometry, such as the one based on nitrogen-vacancy centers [39], or the combination of electron paramagnetic resonance with scanning tunneling microscopy [40] may, in addition, be able to provide an accurate spatial resolution of the spin polarization.

\section{ACKNOWLEDGMENTS}

The authors thank D. Loss and J. Klinovaja for useful discussions. We acknowledge funding from the Spanish Ministerio de Ciencia, Innovacion y Universidades (MICINN; Projects No. FIS2016-79464-P and No. FIS2017-82804P). I.V.T. acknowledges support from Grupos Consolidados UPV/EHU del Gobierno Vasco (Grant No. IT124919). F.S.B. acknowledges funding from the EU's Horizon
2020 innovation program under Grant Agreement No. 800923 (SUPERTED).

\section{APPENDIX A: THE SPIN CURRENT IN THE QUASICLASSICAL APPROACH AT $h=\Delta$}

In this Appendix we derive expressions (18) and (19). Our starting point is Eq. (17), where we first express all energies in units of $\Delta$ (we do not add new notation for the dimensionless variables):

$$
J=\frac{a}{\pi} \int d \omega \operatorname{Im}\left[\frac{\omega^{2}+1+i R}{R \sqrt{\omega^{2}+1-h^{2}-a^{2}+2 i R}}\right],
$$

with $R=\sqrt{h^{2} \omega^{2}+a^{2}\left(\omega^{2}+1\right)}$ and $\alpha k_{F} / \Delta=a$. Keeping only leading-order terms in $a$, we can approximate the integrand

$$
J \approx \frac{2 a}{\pi} \int_{0}^{\infty} d \omega \operatorname{Im}\left[\frac{1}{\sqrt{2 i} R^{\frac{3}{2}}}\right] .
$$

With $a \ll 1$ we can approximate $R \approx \sqrt{\omega^{2}+a^{2}}$; then the integrand becomes

$$
\begin{aligned}
J & \approx-\frac{\sqrt{a}}{\pi} \int_{0}^{\infty} \frac{d \omega}{a} \frac{1}{\left[\left(\omega^{2} / a^{2}\right)+1\right]^{\frac{3}{4}}} \\
& =-2 \sqrt{\frac{a}{\pi}} \frac{\Gamma(5 / 4)}{\Gamma(3 / 4)} .
\end{aligned}
$$

The last equation corresponds to the result (18) in the main text.

For finite temperature the integral Eq. (A1) converts into a sum over Matsubara frequencies,

$$
J=2 a T \sum_{n} \operatorname{Im} \frac{\omega_{n}^{2}+1+i R}{R \sqrt{\omega_{n}^{2}+1-h^{2}-a^{2}+2 i R}} .
$$

At $h=1$ one approximates the sum in the leading order in $a \ll 1, T$ as

$$
\begin{aligned}
J & \approx 2 a T \sum_{n=0}^{\infty} \operatorname{Im} \frac{\Delta^{2}}{\sqrt{2 i} R^{3 / 2}}=-a T \sum_{n=0}^{\infty} \frac{1}{\left(\omega_{n}^{2}\right)^{3 / 4}} \\
& =-\frac{a}{\pi^{3 / 2}} \sqrt{\frac{1}{T}} \sum_{n=0}^{\infty} \frac{1}{|2 n+1|^{3 / 2}} .
\end{aligned}
$$

The last expression corresponds to Eq. (19) in the main text.

\section{APPENDIX B: SOLUTION OF THE EILENBERGER EQUATION FOR A SEMI-INFINITE WIRE}

Here we derive Eqs. (26) and (27) of the main text. The Eilenberger equation in one dimension for the Zeeman field and Rashba SOC oriented as in the main work is

$$
\pm v_{F} \partial_{x} \check{g}_{ \pm}= \pm i \alpha k_{F} \hat{\sigma}^{y}+i \tau_{3}\left(i \omega_{n}+h \hat{\sigma}^{z}\right)+i \tau_{2} \Delta, \check{g}_{ \pm} .
$$

Here $\check{g}_{ \pm}$denotes the Green's functions for both propagation directions $\pm v_{F}$. We assume that the wire extends over the region $x>0$. This equation has to be solved together with the boundary conditions (i) total reflection at $x=0, \breve{g}_{+}(0)=$ $\check{g}_{-}(0)$ and (ii) boundness at $x=+\infty$. 
We seek a perturbative solution of Eq. (24) up to first order in $h$. We make the ansatz

$$
\check{g}_{ \pm}(x)=\check{g}^{(0)}+\check{g}_{ \pm}^{(1)}(x),
$$

where $\check{g}^{(0)}=g_{0} \tau_{3}+f_{0} \tau_{2}$ is the BCS Green's function in Matsubara frequency space, with $g_{0}=\omega_{n} / E, f_{0}=\Delta / E$, and $E=\sqrt{\omega_{n}^{2}+\Delta^{2}} \cdot \breve{g}^{(1)}(x)$ is the first-order correction in the Zeeman field $h$. The first order for correction is obtained from the Eilenberger equation

$$
\partial_{x} g_{ \pm}^{(1)}+\left[ \pm E \check{g}^{(0)}-i \tilde{\alpha} \hat{\sigma}^{y}, \check{g}_{ \pm}^{(1)}\right]= \pm 2 \tau_{1} \hat{\sigma}^{z} h f_{0},
$$

where we set $v_{F}=1$ and $\tilde{\alpha}=\alpha m$. We search for the solution of the form

$$
g_{ \pm}^{(1)}(x)=U_{ \pm}(x) \tilde{g}_{ \pm}(x) U_{ \pm}^{-1}(x)
$$

with $U_{ \pm}=\exp \left(\mp \check{g}^{(0)} E x+i \tilde{\alpha} \hat{\sigma}^{y}\right)$. Inserting this ansatz into Eq. (24) gives

$$
\partial_{x} \tilde{g}^{ \pm}= \pm 2 h f_{0} U_{ \pm}^{-1} \tau_{1} \hat{\sigma}^{z} U_{ \pm}= \pm 2 h f_{0} \tau_{1} \hat{\sigma}^{z} U_{ \pm}^{2} .
$$

Integrating the equation leads to

$$
\begin{aligned}
\tilde{g}_{ \pm}(x)= & \tilde{g}_{ \pm}(0) \pm 2 h f_{0} \tau_{1} \hat{\sigma}^{z} \int_{0}^{x} d x^{\prime} e^{\mp 2 \breve{g}^{(0)} E x^{\prime}} e^{2 i \tilde{\alpha} \hat{\sigma}^{y} x^{\prime}} \\
= & \tilde{g}_{ \pm}(0) \pm \frac{2 h f_{0}}{2\left(E^{2}+\tilde{\alpha}^{2}\right)} \tau_{1} \hat{\sigma}^{z} \\
& \times\left(i \tilde{\alpha} \sigma_{y} \pm E \check{g}^{(0)}\right)\left[1-U_{ \pm}^{2}(x)\right] .
\end{aligned}
$$

With this the solution can be written as

$$
\begin{aligned}
\check{g}_{ \pm}^{(1)}(x)= & \tilde{g}_{ \pm}(0) U_{ \pm}^{-2} \pm \frac{h f_{0}}{E^{2}+\tilde{\alpha}^{2}} \tau_{1} \sigma_{z} \\
& \times\left(i \tilde{\alpha} \sigma_{y} \pm E \check{g}^{(0)}\right)\left(U_{ \pm}^{-2}-1\right)
\end{aligned}
$$

where we used $e^{\check{g}^{(0)} E x} \tilde{g}(0)=\tilde{g}(0) e^{-\breve{g}^{(0)} E x}$ due to the normalization condition of the Green's function. We need to impose boundedness of the solution. So $\tilde{g}(0)$ has to cancel exponentially growing terms in the second term of Eq. (B7). Keeping only exponentially large terms, at $x \rightarrow \infty, U_{ \pm}^{-2}=\frac{1}{2} e^{2 E x}(1 \pm$ $\left.\check{g}^{(0)}\right) e^{-2 i \tilde{\alpha} \hat{\sigma}^{y} x}$, and

$$
\begin{aligned}
\check{g}_{ \pm}^{(1)}\left(x_{\infty}\right)= & \tilde{g}(0) U_{ \pm}^{-2} \\
& \pm \frac{h f_{0}}{E^{2}+\tilde{\alpha}^{2}} \tau_{1} \hat{\sigma}^{z}\left(i \tilde{\alpha} \hat{\sigma}^{y} \pm E \check{g}^{(0)}\right) U_{ \pm}^{-2}
\end{aligned}
$$

We make the ansatz $\tilde{g}(0)=-\frac{h f_{0}}{E^{2}+\tilde{\alpha}^{2}} \tau_{1}\left(E \check{g}^{(0)} \hat{\sigma}^{z}+\tilde{\alpha} b \hat{\sigma}^{x}\right)$, where $b$ has to be determined from the boundary condition at infinity. From Eq. (B8) we obtain

$$
(-b \pm 1)\left(1 \pm \check{g}^{(0)}\right) \frac{1}{2} e^{2 E x} e^{-2 i \tilde{\alpha} \hat{\sigma}^{y} x}=0,
$$

which is solved by $b=\check{g}^{(0)}$. Now we construct the full $\check{g}^{(1)}(x)$ from Eq. (B7). Writing $U_{ \pm}^{-1}=u_{ \pm}^{-1} A^{-1}$, with $A^{-1}=e^{-i \tilde{\alpha} \hat{\sigma}^{y} x}$ and $u_{ \pm}^{-1}=e^{ \pm \check{g}^{(0)} E x}$, we get

$$
\begin{aligned}
\check{g}_{ \pm}^{(1)}(x)= & \frac{h f_{0}}{E^{2}+\tilde{\alpha}{ }^{2}}\left\{\tilde{\alpha} \hat{\sigma}^{x} A^{-2} \tau_{1}\left[-\check{g}^{(0)} \pm 1\right] u_{ \pm}^{-2}\right. \\
& \left.+\left[\mp \tilde{\alpha} \hat{\sigma}^{x}+E \check{g}^{(0)} \hat{\sigma}^{z}\right] \tau_{1}\right\} .
\end{aligned}
$$

The exponentially growing part gets canceled by construction, so we can keep only the decaying part of $u_{ \pm}^{-2}$, which is $u_{ \pm}^{-2}=$ $\frac{1}{2} e^{-2 E x}\left(1 \mp \check{g}^{(0)}\right)$. Inserting this in the above equation finally leads to

$$
\begin{aligned}
\check{g}_{ \pm}^{(1)}(x)= & \frac{h f_{0}}{E^{2}+\tilde{\alpha}^{2}}\left\{\left[\mp \tilde{\alpha} \hat{\sigma}^{x}+E \check{g}^{(0)} \hat{\sigma}^{z}\right]\right. \\
& \left.+\alpha e^{-2 E x} \hat{\sigma}^{x} e^{-2 i \hat{\sigma}^{y} x}\left[\check{g}^{(0)} \pm 1\right]\right\} \tau_{1} .
\end{aligned}
$$

The symmetric part of the above Green's function is

$$
\begin{aligned}
\delta \check{g}_{s}^{(1)}(x) & =\check{g}_{+}^{(1)}(x)+\check{g}_{-}^{(1)}(x) \\
& =2 \frac{h f_{0}}{E^{2}+\tilde{\alpha}^{2}} \check{g}^{(0)} \tau_{1}\left[E \hat{\sigma}^{z}+\tilde{\alpha} \hat{\sigma}^{x} e^{-2 E x} e^{-i 2 \tilde{\alpha} x \check{\sigma}^{y}}\right]
\end{aligned}
$$

The corresponding correction to the magnetization is obtained from $\delta M_{j}=\pi i T \mu_{B} N_{0} \frac{1}{8} \sum_{\omega_{n}} \operatorname{Tr} \tau_{3} \hat{\sigma}^{j} g_{s}^{(1)}$.
[1] I. Žutić, J. Fabian, and S. Das Sarma, Rev. Mod. Phys. 76, 323 (2004).

[2] E. I. Rashba, in Future Trends in Microelectronics: Up the Nano Creek, edited by S. Luryi, J. M. Xu, and A. Zaslavsky (Wiley, Hoboken, NJ, 2007), pp. 28-40.

[3] Spin Current, edited by S. Maekawa, S. O. Valenzuela, E. Saitoh, and T. Kimura (Oxford University Press, Oxford, 2012).

[4] M. Eschrig, Phys. Today 64(1), 43 (2011).

[5] E. I. Rashba, Phys. Rev. B 68, 241315(R) (2003).

[6] J. Shi, P. Zhang, D. Xiao, and Q. Niu, Phys. Rev. Lett. 96, 076604 (2006).

[7] J. Wang and K. S. Chan, Phys. Rev. B 74, 035342 (2006).

[8] E. B. Sonin, Phys. Rev. B 76, 033306 (2007).

[9] E. B. Sonin, Phys. Rev. Lett. 99, 266602 (2007).

[10] H.-A. Engel, E. I. Rashba, and B. I. Halperin, in Handbook of Magnetism and Advanced Magnetic Materials, edited by $\mathrm{H}$.
Kronmüller and S. Parkin (Wiley, New York, 2007), Vol. 5, p. 2858.

[11] F. J Jedema, H. B. Heersche, A. T. Filip, J. Baselmans, and B. Wees, Nature (London) 416, 713 (2002).

[12] H. Nakayama, M. Althammer, Y. T. Chen, K. Uchida, Y. Kajiwara, D. Kikuchi, T. Ohtani, S. Geprägs, M. Opel, S. Takahashi et al., Phys. Rev. Lett. 110, 206601 (2013).

[13] I. V. Tokatly, Phys. Rev. Lett. 101, 106601 (2008).

[14] I. V. Tokatly and E. Sherman, Ann. Phys. (NY) 325, 1104 (2010).

[15] F. Dolcini and F. Rossi, Phys. Rev. B 98, 045436 (2018).

[16] A. A. Abrikosov and L. P. Gor'kov, Zh. Eksp. Teor. Fiz. 42, 1088 (1962)[Sov. Phys. JETP. 15, 752 (1962)].

[17] A. A. Abrikosov, Fundamentals of the Theory of Metals (North Holland, Amsterdam, New York, 1988).

[18] L. P. Gor'kov and E. I. Rashba, Phys. Rev. Lett. 87, 037004 (2001). 
[19] J. D. Sau, R. M. Lutchyn, S. Tewari, and S. Das Sarma, Phys. Rev. Lett. 104, 040502 (2010).

[20] Y. Oreg, G. Refael, and F. von Oppen, Phys. Rev. Lett. 105, 177002 (2010).

[21] V. Mourik, K. Zuo, S. M. Frolov, S. R. Plissard, E. P. A. M. Bakkers, L. P. Kouwenhoven, Science 336, 1003 (2012).

[22] A. Das, Y. Ronen, Y. Most, Y. Oreg, M. Heiblum, and H. Shtrikman, Nat. Phys. 8, 887 (2012).

[23] M. T. Deng, C. L. Yu, G. Y. Huang, M. Larsson, P. Caroff, and H. Q. Xu, Nano Lett. 12, 6414 (2012).

[24] M. T. Deng, S. Vaitiekenas, E. B. Hansen, J. Danon, M. Leijnse, K. Flensberg, J. Nygård, P. Krogstrup, and C. M. Marcus, Science 354, 1557 (2016).

[25] D. Sticlet, C. Bena, and P. Simon, Phys. Rev. Lett. 108, 096802 (2012).

[26] T. Ojanen, Phys. Rev. Lett. 109, 226804 (2012).

[27] J. Tiira, E. Strambini, M. Amado, S. Roddaro, P. San-Jose, R. Aguado, F. S. Bergeret, D. Ercolani, L. Sorba, and F. Giazotto, Nat. Commun. 8, 14984 (2017).

[28] J. Paajaste, M. Amado, S. Roddaro, F. S. Bergeret, D. Ercolani, L. Sorba, F. Giazotto, Nano Lett. 15, 1803 (2015).

[29] H. Zhang, Ö. Gül, S. Conesa-Boj, M. P. Nowak, M. Wimmer, K. Zuo, V. Mourik, F. K. de Vries, J. van Veen, and M. W. A. de Moor, J. D. S. Bommer et al., Nat. Commun. 8, 16025 (2017).
[30] W. Chang, S. M. Albrecht, T. S. Jespersen, F. Kuemmeth, P. Krogstrup, J. Nygård, and C. M. Marcus, Nat. Nanotechnol. 10, 232 (2015).

[31] R. Lutchyn, E. P. A. M. Bakkers, L. P. Kouwenhoven, P. Krogstrup, C. M. Marcus, and Y. Oreg, Nat. Rev. Mater. 3, 52 (2018).

[32] P. Szumniak, D. Chevallier, D. Loss, and J. Klinovaja, Phys. Rev. B 96, 041401(R) (2017).

[33] K. Björnson, S. S. Pershoguba, A. V. Balatsky, and A. M. BlackSchaffer, Phys. Rev. B 92, 214501 (2015).

[34] M. Serina, D. Loss, and J. Klinovaja, Phys. Rev. B 98, 035419 (2018).

[35] Notice that expression (6) makes a connection between the bulk spin current and the total transverse spin. The spatial distribution of the latter has to be computed by solving the full boundary value problem. In a normal wire this was done in Ref. [15].

[36] G. Eilenberger, Z. Phys. 214, 195 (1968).

[37] F. Konschelle, I. V. Tokatly, and F. S. Bergeret, Phys. Rev. B 92, 125443 (2015).

[38] I. V. Tokatly, Phys. Rev. B 96, 060502(R) (2017).

[39] F. Casola, T. van der Sar, and A. Yacoby, Nat. Rev. Mater. 3, 17088 (2018).

[40] S. Baumann, W. Paul, T. Choi, C. P. Lutz, A. Ardavan, and A. J. Heinrich, Science 350, 417 (2015). 